\title{
Literacia em saúde e o autocuidado e autocontrolo no idoso com diabetes tipo $2^{*}$
}

\author{
Health Literacy and Self-Care and Self-Monitoring in the Elderly with Type 2 Diabetes \\ Alfabetización en salud y en el autocuidado y autocontrol en adulto mayor con diabetes tipo 2
}

Maria Edite Rua Castro ${ }^{\text {a }}$

ACES Cávado III Barcelos/Esposende, Portugal

DOI: https://doi.org/10.11144/Javeriana.ie22.lsaa

mecastro72@hotmail.com

ORCID: https://orcid.org/0000-0001-9701-828X

Recepção: 07 Julho 2019

Aprovação: 19 Maio 2020

Salete Soares

Publicação: 29 Dezembro2020

Escola Superior de Saúde, Portugal

ORCID: https://orcid.org/0000-0003-3582-044X

\section{Resumo:}

Introdução. A diabetes como doença crónica, representa um grave problema de saúde pública a nível mundial, pela sua incidência, e pela elevada taxa de morbilidade e mortalidade com fortes repercussões na vida das pessoas. Objetivo. Conhecer efeito de programa de educação para a saúde sobre o conhecimento da diabetes, capacitação para o autocuidado e literacia em saúde no idoso diabético. Método. Estudo quasi-experimental de grupo único, com amostra de 40 participantes diabéticos tipo 2, com 65 ou mais anos de idade, autónomos. Instrumentos: Questionário Sociodemográfico e Clínico; Questionário de Avaliação de Conhecimentos (DKQ-24) $(\alpha=0,83)$; Escala Atividades de Autocuidado com a Diabetes $(\alpha=0,62)$; Questionário Literacia Newest Vital Sign $(\alpha=0,76)$. Realizou-se uma análise estatística descritiva e inferencial. Resultados. Diferenças estatisticamente significativas no conhecimento sobre diabetes entre o $1^{\circ} \mathrm{e} 2^{\circ}$ momento de avaliação. Nas atividades de autocuidado com a diabetes encontramos diferenças estatisticamente significativas nas atividades Alimentação, Alimentação Específica e Cuidados com os Pés. A literacia em saúde, na $1^{a}$ avaliação, situou-se a um nível de alta probabilidade de literacia limitada e após intervenção evoluiu positivamente, demonstrando um aumento do nível de literacia. Conclusão. Este estudo comprovou a importância dos programas de educação para a saúde como uma ferramenta potente para a capacitação da pessoa diabética. Verificou-se que o nível de conhecimentos sobre a doença na pessoa diabética tipo 2 com mais de 65 anos aumentou de forma significativa, e interferiu positivamente na capacitação do diabético idoso para a gestão das atividades de autocuidado aumentando o nível de literacia em saúde.

Palavras-chave: literacia em saúde, autocuidado, diabetes mellitus, autocontrolo, doença crónica.

\section{Abstract:}

Introduction. Diabetes as a chronic disease, represents a serious public health problem worldwide, due to its incidence, and the high rate of morbidity and mortality with strong repercussions in people's lives. Objective. To know the effect of a health education program on the knowledge of diabetes, self-care training and health literacy in the elderly diabetic. Method. Quasiexperimental study of a single group, with a sample of 40 type 2 diabetic participants, aged 65 or over, autonomous. Instruments: Sociodemographic and Clinical Questionnaire; Knowledge Assessment Questionnaire (DKQ-24) $(\alpha=0,83)$; Summary of Diabetes Self-Care Activities $(\alpha=0,62)$; Literacy Questionnaire Newest Vital Sign $(\alpha=0,76)$. A descriptive and inferential statistical analysis was conducted. Results. Statistically significant differences identified in the knowledge about diabetes between the 1st and 2nd assessment. Regarding self-care activities with diabetes, statistically significant differences were found in the activities Diet, Specific Diet and Foot Care. Health literacy, in the 1st evaluation, was at a high probability level of limited literacy and after intervention evolved positively, showing an increase in the level of literacy. Conclusion. This study proved the importance of health education programs, as a powerful tool for self-care training the diabetic person. It was confirmed that the level of knowledge about the disease in the type 2 diabetic person over 65 years of age increased significantly, but also contributed positively in the diabetic's capacity to manage self-care activities and increased the level of health literacy.

Keywords: health literacy, self-care, diabetes mellitus, self-monitoring, chronic desease.

\section{Resumen:}

Introducción. La diabetes como enfermedad crónica, representa un grave problema de salud pública a nivel mundial, por su incidencia y por la alta tasa de morbilidad y mortalidad con fuertes repercusiones en la vida de las personas. Objetivo. Conocer

\section{Autor notes}

a Autora correspondente. Correio eletrônico: mecastro72@hotmail.com 
el efecto del programa de educación para la salud sobre el conocimiento de la diabetes, capacitación para el autocuidado y alfabetización en salud en el adulto mayor diabético. Método. Estudio cuasi-experimental de grupo único, con una muestra de 40 participantes diabéticos tipo 2, de 65 o más años de edad, independientes. Instrumentos: Cuestionario sociodemográfico y clínico; Cuestionario de evaluación de conocimientos (DKQ-24) $(\alpha=0,83)$; Escala Actividades de Autocuidado con la Diabetes $(\alpha=0,62)$; Cuestionario Alfabetización Newest Vital Sign $(\alpha=0,76)$. Se realizó un análisis estadístico descriptivo e inferencial. Resultados. Diferencias estadísticamente significativas en el conocimiento sobre diabetes entre el ler y el 2 do momento de evaluación. En las actividades de autocuidado con la diabetes encontramos diferencias estadísticamente significativas en las actividades Alimentación, Alimentación Específica y Cuidados de los Pies. La alfabetización en salud, en la $1^{\text {a }}$ evaluación, se situó en un nivel de alta probabilidad de alfabetización limitada y después de la intervención evolucionó positivamente, demostrando un aumento en el nivel de alfabetización. Conclusión. Este estudio comprobó la importancia de los programas de educación para la salud como herramienta potente para la capacitación de la persona diabética. Se verificó que el nivel de conocimientos sobre la enfermedad en la persona diabética tipo 2 mayor de 65 años aumentó de forma significativa e interfirió positivamente en la capacitación del diabético adulto mayor para la gestión de las actividades de autocuidado aumentando el nivel de alfabetización en salud.

Palabras clave: alfabetización en salud, autocuidado, diabetes mellitus, autocontrol, enfermedad crónica.

\section{Introdução}

A diabetes sendo uma doença metabólica afeta um grande número de pessoas, devido à sua cronicidade e elevada morbilidade, originando um enorme investimento em cuidados de saúde, resultante de elevados custos humanos, económicos e sociais, tornando-se um dos maiores problemas de saúde pública mundiais. Perante este cenário, diversos esforços têm sido desenvolvidos por parte de governantes e nomeadamente grupos científicos para evitar o desenvolvimento da doença.

A incidência da diabetes tem vindo a aumentar nos últimos anos, nomeadamente tipo 1 e tipo 2, tendo contribuído para este facto os fatores genéticos, ambientais e comportamentais, entre os quais uma alimentação altamente calórica e o sedentarismo, sendo os maiores contribuidores para a obesidade, apesar de haver mais cuidados na sua prevenção, diagnóstico precoce e dos progressos terapêuticos farmacológicos (1).

Em Portugal cerca de 19\% da população com mais de 65 anos de idade e mais de um quarto da população portuguesa referente ao escalão dos 60-79 anos têm diabetes $(1,2)$. Tendo como referência os dados epidemiológicos da diabetes, torna-se de extrema importância a literacia em saúde e empoderamento dos cidadãos, nomeadamente, das pessoas com esta patologia, no sentido da manutenção de uma vida com qualidade.

A diabetes tem uma prevalência superior na faixa etária dos 65 ou mais anos. "A Diabetes Melitus aumenta com a idade, atingindo valores mais elevados no grupo dos 65 aos 74 anos. Em Portugal aproximadamente metade dos diabéticos conhecidos tem mais de 65 anos de idade" (3). "Os diabéticos idosos recorrem com muita mais frequência aos serviços de saúde, consomem mais medicamentos e tem uma morbilidade e mortalidade mais alta do que o resto da população" (4). Associado ao aumento da idade, surgem as complicações como a retinopatia e a neuropatia diabética. Tudo isto se passa numa altura da vida em que as pessoas estão mais limitadas nas suas capacidades, mais dependentes de terceiros e têm também, com frequência, outras doenças associadas. A única forma de mudar estas perspetivas é fazer um diagnóstico precoce e estabelecer um bom controlo da diabetes e dos fatores de risco. Os diabéticos bem controlados têm melhor qualidade de vida, recorrem menos aos centros de saúde e têm menos hospitalizações (4).

Assim, torna-se necessário investir na literacia em saúde junto deste grupo de pessoas para alterar este trajeto e transforma-lo em saúde com mais qualidade e minimizar custos, justificando-se a criação de estratégias facilitadoras de uma melhor compreensão da informação e que permitam às pessoas com baixa literacia, ultrapassar obstáculos e alcançar objetivos positivos face ao regime terapêutico.

O tratamento e a prevenção de complicações, só se tornará eficaz quando houver um real empenho do próprio doente, uma vez que depende do mesmo a tomada de decisão e consequentemente mudança nos hábitos quotidianos. 


\section{Objetivo}

Este estudo tem como objetivo geral conhecer o efeito de um programa de educação para a saúde sobre o conhecimento da diabetes, capacitação para o autocuidado e literacia em saúde no idoso diabético, tendo como finalidade contribuir para melhorar a sua qualidade de vida e para alcançar com eficácia ganhos em saúde.

\section{Método}

A opção metodológica centrou-se num estudo quase-experimental com desenho antes-após, de grupo único. O termo antes refere-se ao momento de avaliação antes da intervenção e o após corresponde ao momento de avaliação depois da intervenção, tendo como variável independente o Programa de Educação para a Saúde ao idoso diabético tipo 2.

Neste estudo optou-se por uma amostra não probabilística por conveniência de pessoas com diabetes tipo 2 de uma Unidade de Cuidados de Saúde Personalizados (UCSP) da região norte do país, constituída por $n=40$ que integram esta UCSP, sendo selecionados com critérios de inclusão idade igual ou superior a 65 anos e de exclusão ser dependente ou acamado.

Pretende-se verificar se $\mathrm{O}$ programa de educação para a saúde aumenta o nível de conhecimentos sobre a doença na pessoa diabética tipo 2, com mais de 65 anos $(\mathrm{H} 1)$ e se $\mathrm{O}$ programa de educação para a saúde capacita as pessoas com diabetes tipo 2, com mais de 65 anos, para gerir as atividades de autocuidado (H2).

O programa tipo de educação para a saúde construído para o efeito teve os seguintes eixos estruturantes: diabetes-fisiopatologia e tratamento; alimentação equilibrada; exercício físico e cuidados com os pés, sendo sempre que necessário ajustado às necessidades de cada participante. Formaram-se grupos de duas pessoas a quem foi realizado uma sessão de educação para a saúde com a duração de cerca de 2 horas, utilizando uma metodologia expositiva, interativa e demonstrativa com recurso pedagógico a diapositivos, brainstorming e debate de ideias.

Recorremos a diferentes instrumentos, nomeadamente a um Questionário Sociodemográfica e Clínico, construído para o efeito, composto por um conjunto de questões, nomeadamente sexo, idade, estado civil, grau de escolaridade, situação profissional, IMC, perímetro abdominal, TA diastólica, TA sistólica, hemoglobina glicada, tipo de tratamento para a diabetes.

O Questionário de Avaliação de Conhecimentos (DKQ-24) (5) que integra várias questões associadas com o conhecimento global da diabetes, tais como: tipo de diabetes, as causas da diabetes, fisiopatologia, tratamentos para a diabetes, sinais e sintomas de hipoglicemia e hiperglicemia, complicações crónicas da diabetes. Tem como respostas possíveis sim, não e não sei, pontuadas 1,0 e 2, respetivamente e que determinam respostas certas, erradas e desconhecimento.

Escala de Atividades de Autocuidado com a Diabetes (6) que mede a frequência das atividades realizadas, nomeadamente, alimentação, atividade física, monitorização de glicemia, cuidados com os pés, medicamentos e hábitos tabágicos.

Esta avaliação é parametrizada por dias de semana numa escala de 0 a 7 , sendo a pontuação zero a menos desejada e a pontuação sete a mais benéfica.

Questionário de Literacia Newest Vital Sign (7) composto por seis questões com interpretação de informação escrita e numérica através de um rótulo nutricional de gelado, correspondendo a cada resposta certa 1 ponto e zero pontos por cada resposta errada. Respostas certas entre $0-1$ questóes significam $50 \%$ ou mais de probabilidade de literacia limitada, entre 2 a 3 indica possibilidade de literacia limitada e superior a 4 a 6 respostas certas indica níveis elevados de literacia em saúde (7). 
Para a colheita de dados, foi criado um questionário de caraterização da amostra e escolhidas três escalas, já traduzidas e validadas para a população portuguesa. Os dados referentes às caraterísticas sociodemográficas e clínicas foram efetuados apenas no $1^{\circ}$ momento de avaliação. Os dados relativos à avaliação dos conhecimentos, às atividades de autocuidado e ao nível de literacia em saúde foram recolhidos antes e após a intervenção de educação para a saúde. Todos os instrumentos foram preenchidos através de entrevista individual pelo investigador, devido ao baixo nível de escolaridade dos idosos.

O tratamento de dados foi efetuado com recurso ao SPSS versão 23 utilizando estatística descritiva e inferencial com um nível de confiança de 95\%.

O estudo da normalidade da amostra foi realizado pelo teste de Shapiro-Wilk $(W)$ quando $n<50$ e a homogeneidade das variâncias foi verificada pelo teste de Levene $(F)$. Para a comparação entre mulheres e homens recorremos ao teste U Mann-Whitney e para a comparação entre os dois momentos de observação ao teste de Wilcoxon devido às variáveis não apresentarem distribuição normal. A consistência interna foi avaliada pelo a-Cronbach.

Foram respeitados todos os princípios éticos de acordo com o Código de Nuremberga, a Declaração de Helsínquia e o Relatório de Belmont e foi obtida aprovação pela Comissão de Ética da Instituição onde se desenvolveu o estudo com o Parecer n 20/2018.

\section{Resultados}

Caraterização sociodemográfica - A amostra era constituída por participantes do sexo feminino (52,5\%) e sexo masculino (47,5\%).

Na tabela 1 que a seguir se apresenta podemos observar a distribuição das variáveis sociodemográficas que caraterizam a amostra.

TABELA 1.

Caracterização Sociodemográfica da Amostra em estudo segundo a Idade, Estado Civil, Grau de Escolaridade, Profissão e Situação Profissional

\begin{tabular}{|c|c|c|c|c|c|c|c|c|c|c|c|}
\hline & \multicolumn{3}{|c|}{ Feminino } & \multicolumn{4}{|c|}{ Masculino } & \multicolumn{4}{|c|}{ Todos } \\
\hline $\mathbf{M}$ & DP & Mín & Máx & M & DP & Mín & Máx & $\mathbf{M}$ & DP & Mín & Máx \\
\hline \multirow[t]{3}{*}{ Idade } & 5,96 & 65 & 86 & 72,1 & 4,19 & 66 & 80 & 72,3 & 5,13 & 65 & 86 \\
\hline & & & & \multicolumn{3}{|c|}{ Feminino } & \multicolumn{3}{|c|}{ Masculino } & \multicolumn{2}{|c|}{ Todos } \\
\hline & & & & & $n$ & $\%$ & $n$ & & $\%$ & $n$ & $\%$ \\
\hline \multicolumn{12}{|l|}{ Estado Civil } \\
\hline Solteiro & & & & 2 & & 9,5 & --- & & --- & 2 & 5 \\
\hline Casado(a) & & & & 10 & & 47,6 & 17 & & 89,5 & 27 & 67,5 \\
\hline Viúvo(a) & & & & 9 & & 42,9 & $-\cdots$ & & $-\cdots$ & 9 & 22,5 \\
\hline Divorciado(a) & & & & $-\cdots$ & & 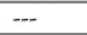 & 2 & & 10,5 & 2 & 5 \\
\hline \multicolumn{12}{|l|}{ Grau de Escolaridade } \\
\hline Não sabe ler nem escrever & & & & 2 & & 9,5 & -- & & $-\cdots$ & 2 & 5 \\
\hline Não completou nenhum g & rau de & ensino & & 7 & & 33,3 & 3 & & 15,8 & 10 & 25 \\
\hline $1^{\text {a }}$ Ciclo do Ensino Básico & & & & 12 & & 57,1 & 12 & & 63,2 & 24 & 60 \\
\hline $2^{\circ}$ Ciclo Ensino Básico-6º & ano & & & $-\cdots$ & & -- & 2 & & 10,5 & 2 & 5 \\
\hline $3^{\circ}$ Ciclo do Ensino Básico & $-9^{\circ}$ an & & & $-\cdots$ & & --- & 1 & & 5,3 & 1 & 2,5 \\
\hline Bacharelato & & & & $-\ldots$ & & $-\cdots$ & 1 & & 5,3 & 1 & 2,5 \\
\hline \multicolumn{12}{|l|}{ Profissảo } \\
\hline Doméstico(a) & & & & 12 & & 57,1 & 1 & & 5,3 & 13 & 32,5 \\
\hline Operário da Construçáo $\mathrm{C}$ & ivil & & & $-\ldots$ & & $-\ldots$ & 5 & & 26,3 & 5 & 12,5 \\
\hline \multicolumn{12}{|c|}{ Situaçáo Profissional } \\
\hline Desempregada & & & & 3 & & 14,3 & $-\cdots$ & & $-\cdots$ & 3 & 7,5 \\
\hline Reformado(a) & & & & 18 & & 85,7 & 19 & & 100 & 37 & 92,5 \\
\hline
\end{tabular}

Fonte: elaboraçao própria (2019)

No que se refere à caraterização clínica salientamos que em relação ao tipo de tratamento para a diabetes, a quase totalidade dos sujeitos recorre a antidiabéticos orais (mulheres: $n=20 ; 95,2 \%$; homens: $n=17 ; 89,5 \%$ ).

A tabela 2 apresenta a distribuição dos valores referentes à caracterização clínica. 
TABELA 2.

Caracterização Clínica da Amostra em estudo segundo o Tipo de Tratamento para a Diabetes, Peso, Altura, Índice de Massa Corporal, Perímetro Abdominal, Tensão Arterial Máxima, Tensão Arterial Mínima, Hemoglobina Glicada (momento 1), Hemoglobina Glicada (Momento 2) e Anos Como Diabético

\begin{tabular}{|c|c|c|c|c|c|c|c|c|c|c|c|c|}
\hline & & & \multicolumn{3}{|c|}{ Feminino } & \multicolumn{3}{|c|}{ Masculino } & \multicolumn{3}{|c|}{ Todos } & \\
\hline & & & & $N$ & $\%$ & & $n$ & $\%$ & $n$ & $\%$ & & \\
\hline \multicolumn{12}{|c|}{$\begin{array}{l}\text { Tipo de Tratamento para } \\
\text { a Diabetes }\end{array}$} & \\
\hline \multirow{2}{*}{\multicolumn{3}{|c|}{$\begin{array}{l}\text { Antidiabéticos Orais } \\
\text { Outro }\end{array}$}} & & 20 & \multicolumn{2}{|c|}{95,2} & 17 & 89,5 & & \multicolumn{2}{|c|}{92,5} & \\
\hline & & & & 1 & \multicolumn{2}{|c|}{4,8} & 2 & 10,5 & \multicolumn{2}{|c|}{3} & 7,5 & \\
\hline & \multicolumn{4}{|c|}{ Feminino } & \multicolumn{4}{|c|}{ Masculino } & \multicolumn{4}{|c|}{ Todos } \\
\hline Variáveis & M & DP & Mín & Máx & M & DP & Mín & Máx & M & DP & Mín & Máx \\
\hline Peso & 71,5 & 10,24 & 52,5 & 95 & 81,4 & 13,77 & 52,5 & 100 & 76,2 & 12,89 & 52,5 & 100 \\
\hline Altura & 156,1 & 4,72 & 150 & 168 & 163,8 & 6,6 & 151 & 173 & 159,8 & 6,83 & 150 & 173 \\
\hline IMC & 29,3 & 4,39 & 21 & 41,4 & 29,9 & 4,26 & 19,1 & 35,1 & 29,6 & 4,29 & 19,1 & 41,4 \\
\hline PA & 107 & 9,46 & 88 & 125 & 108,8 & 11,12 & 87 & 124 & 107,9 & 10,19 & 87 & 125 \\
\hline TA-Max & 149,5 & 18,8 & 120 & 208 & 144,1 & 19,23 & 112 & 170 & 146,9 & 18,97 & 112 & 208 \\
\hline TA-Min & 77 & 10,2 & 56 & 94 & 71,9 & 10,27 & 55 & 90 & 74,6 & 10,41 & 55 & 94 \\
\hline HG 1 & 7,1 & 1,61 & 5,2 & 12,8 & 6,6 & 0,92 & 5,3 & 8,6 & 6,9 & 1,34 & 5,2 & 12,8 \\
\hline HG 2 & 7 & 1,46 & 5,3 & 10 & 6,5 & 0,84 & 5,1 & 8 & 6,8 & 1,21 & 5,1 & 10 \\
\hline $\begin{array}{l}\text { Anos Como } \\
\text { Diabético(a) }\end{array}$ & 10,5 & 4,54 & 3 & 20 & 9,5 & 4,89 & 1 & 20 & 10,1 & 4,67 & 1 & 20 \\
\hline
\end{tabular}

Fonte: elaboraçao própria (2019)

A tabela 3 apresenta os resultados da comparação entre os dois momentos (momento 1 e momento 2) em cada grupo. Constata-se que existe evidência de diferenças estatisticamente significativas na variável "respostas corretas" quer nas mulheres como nos homens, no que se refere aos conhecimentos sobre diabetes.

Nas atividades de autocuidado com a diabetes constatamos que nas variáveis "alimentação", "alimentação específica" e "cuidado com os pés" existem diferenças estatisticamente significativas quer nas mulheres como nos homens, demonstrativas da evolução positiva nestas atividades de autocuidado.

TABELA 3.

Comparação dos Valores por Grupo e por Momento de Avaliação dos Conhecimentos sobre a Diabetes e Atividades de Autocuidado com a Diabetes

\begin{tabular}{|c|c|c|c|c|c|c|c|c|}
\hline & \multicolumn{4}{|c|}{ Feminino } & \multicolumn{4}{|c|}{ Masculino } \\
\hline & \multicolumn{8}{|c|}{ Teste de Wilcoxon } \\
\hline & $\begin{array}{c}\text { Ranks } \\
\text { negativos }\end{array}$ & $\begin{array}{c}\text { Ranks } \\
\text { positivos }\end{array}$ & $z$ & $p$ & $\begin{array}{c}\text { Ranks } \\
\text { negativos }\end{array}$ & $\begin{array}{c}\text { Ranks } \\
\text { positivos }\end{array}$ & $Z$ & $p$ \\
\hline \multicolumn{9}{|l|}{ CSD } \\
\hline Resp. Corretas & 2 & 11,45 & $-3,951$ & $\begin{array}{l}<0 \\
1^{*}\end{array}$ & 0 & $10 \quad 0$ & $-3,834$ & $<0 \quad 1^{*}$ \\
\hline \multicolumn{9}{|l|}{ AACD } \\
\hline Alimentaçáo & 1 & 8 & $-3,235$ & $0,001^{*}$ & 0 & 8,5 & $-3,524$ & $\begin{array}{ll}<0 & 1^{*}\end{array}$ \\
\hline $\begin{array}{l}\text { Alimentação } \\
\text { específica }\end{array}$ & 4,2 & 11,54 & $-2,813$ & $0,005^{*}$ & 2,63 & 11,97 & $-3,403$ & $0,001^{*}$ \\
\hline Atividade física & 3,33 & 5,2 & $-1,124$ & 0,261 & 4,67 & 5,17 & $-1,021$ & 0,307 \\
\hline Mon. Glicémia & 9,5 & 6,39 & $-0,317$ & 0,752 & 4,33 & 6 & $-1,493$ & 0,136 \\
\hline Cuidado cl pés & 0 & 4,5 & $-2,524$ & $0,012^{*}$ & 0 & 6 & $-2,940$ & $0,003^{*}$ \\
\hline Medicamentos & 0 & 1 & -1 & 0,317 & 1 & 2 & $-0,477$ & 0,655 \\
\hline
\end{tabular}

Nota: CSD: Conhecimentos sobre diabetes; AACD: Atividades de autocuidado com a diabetes; ${ }^{*}$ diferenças estatisticamente significativas $(\mathrm{p}<0,05)$ Fonte: elaboraçao própria (2019)

Os resultados evidenciam a aquisição de conhecimentos sobre diabetes e a adoção de comportamentos de autocuidado com a diabetes.

$\mathrm{Na}$ tabela 4 são apresentados os resultados, que evidenciam uma melhoria do nível de literacia quer nas mulheres, quer nos homens.

No $1^{\circ}$ momento de avaliação em ambos os sexos a grande maioria das respostas situavam-se em " $50 \%$ ou mais de probabilidade de literacia limitada”, evoluindo maioritariamente para respostas classificadas com "possibilidade de literacia limitada". 
TABELA 4.

Níveis de literacia em Saúde. Distribuição dos Valores por Sexo no $1^{\circ}$ e $2^{\circ}$ momento

\begin{tabular}{lllll}
\hline & \multicolumn{2}{c}{ Mulheres (n21) } & \multicolumn{2}{c}{ Homens (n19) } \\
\hline & Momento 1 & Momento 2 & Momento 1 & Momento 2 \\
\hline $\begin{array}{l}\text { 0-1 Pontos sugere alta } \\
\text { probabilidade (50\% ou } \\
\text { mais) de literacia }\end{array}$ & $\begin{array}{l}n=17 \\
(81 \%)\end{array}$ & $n=4(19 \%)$ & $\begin{array}{l}n=12 \\
(63,1 \%)\end{array}$ & $n=3(15,8 \%)$ \\
limitada & & & $n=4$ & $n=11$ \\
\hline $\begin{array}{l}\text { 2-3 Pontos indica a } \\
\text { possibilidade de literacia } \\
\text { limitada }\end{array}$ & $n=4(19 \%)$ & $\begin{array}{l}n=14 \\
(66,7 \%)\end{array}$ & $\begin{array}{l}(21,1 \%) \\
(5-67,9 \%)\end{array}$ \\
\hline $\begin{array}{l}\text { 4-6 Pontos quase } \\
\text { sempre indica literacia } \\
\text { adequada }\end{array}$ & --- & $\begin{array}{l}n=3 \\
(14,3 \%)\end{array}$ & $\begin{array}{l}n=3 \\
(15,8 \%)\end{array}$ & $n=5(26,3 \%)$ \\
\hline
\end{tabular}

Fonte: elaboraçao própria (2019)

Verificou-se, ainda, que nas mulheres no $1^{\circ}$ momento nenhuma se situava no "nível literacia em saúde adequada", mas no $2^{\circ}$ momento $14,3 \%$ - 3 mulheres situam-se neste nível. Relativamente aos homens $15,8 \%$ - 3 situavam-se neste nível no $1^{\circ}$ momento, mas este valor foi mais elevado no $2^{\circ}$ momento $26,3 \%$ - 5 .

\section{Discussão}

A maioria dos participantes têm um peso acima do considerado ideal, sendo a média de IMC $29,6 \mathrm{~kg} / \mathrm{m}^{2}$, compatível com uma pré-obesidade e apresenta-se como um fator determinante para a evolução da DM e dificultador do tratamento e controlo da diabetes. Existe uma forte relação entre o escalão de IMC e a diabetes, com cerca de $90 \%$ da população com diabetes a apresentar excesso de peso (1), também, o mesmo acontece relativamente ao perímetro abdominal com valores médios de $107 \mathrm{~cm}$ para as mulheres e $108,8 \mathrm{~cm}$ para os homens, que se traduz por um desequilíbrio grave em relação à sua condição nutricional, sendo a obesidade um fator de risco para o desenvolvimento da diabetes $(8,9,4)$.

Recomenda-se valores de $\mathrm{HbA} 1 \mathrm{c} \leq 6,5 \%$ (10), estando os participantes do sexo masculino, próximos destes valores, contrariando deste modo, outro estudo (11) tendo concluído que $100 \%$ da sua amostra não alcançou estas metas.

Relativamente ao nível de conhecimentos sobre a diabetes verificaram-se diferenças estatisticamente significativas $(p=0,001)$ pelo que se confirma a Hipótese 1 - O programa de educação para a saúde aumenta

o nível de conhecimentos sobre a doença na pessoa diabética tipo 2, com mais de 65 anos.

No que se refere à Hipótese 2 - O programa de educação para a saúde capacita as pessoas com diabetes tipo 2 com mais de 65 anos, para a gestão das atividades de autocuidado, esta apenas foi confirmada em parte, uma vez que no autocuidado atividade física, monitorização da glicémia e medicamentos não foram encontradas diferenças estatisticamente significativas nestes indicadores de saúde entre o $1^{\circ}$ e o $2^{\circ}$ momento de avaliação.

Da análise dos resultados podemos constatar que a intervenção de enfermagem no âmbito da educação para a saúde resulta em evolução positiva na área do conhecimento sobre diabetes e nas atividades de autocuidado com a diabetes, embora pareça ser necessário que esta intervenção seja mais alargada no tempo de modo a ter efeito em todas as variáveis.

Reconhecendo a relevância e influência da Literacia em Saúde na DM, torna-se assim fundamental que esta seja adequada à pessoa portadora desta doença para uma melhor autogestão da mesma. Nos resultados deste estudo no momento 1 de avaliação predominam respostas classificadas como "alta probabilidade ( $50 \%$ ou mais) de literacia limitada" (mulheres $n=17(81 \%)$ e os homens $n=12(63,1 \%)$, estando em consonância com o nível de literacia em saúde na população portuguesa em geral $(12,13)$. No momento 2 de avaliação após a intervenção de educação para a saúde, surge uma evolução maioritariamente para respostas classificadas como "possibilidade de literacia limitada" (mulheres $n=14(66,7 \%)$ e homens $n=11(57,9 \%)$.

Assim, a educação para a saúde integra uma ferramenta importante na prática clínica dos enfermeiros, pois vai possibilitar responsabilidades partilhadas entre o utente e o enfermeiro (14), envolve uma transmissão 
real, estruturada e organizada de aptidões do educador para o utente, com o objetivo de progressivamente, modificar a dependência da doença em responsabilidade e parceria com a equipa multidisciplinar. "É necessário educar as pessoas providenciando-lhes ferramentas e skills para gerir a sua própria doença, tornando-os autónomos, capacitando-os" (14).

Consideramos que numa perspetiva de continuidade esta intervenção poderá dar mais contributos e que se reveste de uma boa estratégia para o controlo da doença, até porque este estudo apresenta alguns condicionalismos e limitações. Consideramos que um dos condicionalismos está associado à elevada faixa etária e à baixa escolaridade dos inquiridos e uma limitação foi o curto período de acompanhamento para avaliação do programa educativo, os quais idealmente devem ser superiores a 12 meses, para melhor avaliar o efeito da intervenção (15), mas o tempo de duração do projeto de investigação com apenas seis meses não é compatível com esta recomendação, pelo que consideramos importante que se façam estudos semelhantes, com um período de tempo de intervenção de pelo menos 12 meses e com amostras de maior dimensão.

\section{Conclusão}

Este estudo teve por base conhecer o efeito de um programa de educação para a saúde sobre o conhecimento da diabetes, capacitação para o autocuidado e literacia em saúde no idoso diabético, com o objetivo de aumentar o nível de conhecimentos sobre a doença, melhorar a capacidade para o autocuidado, aumentar os níveis de literacia em saúde, e desta forma melhorar o autocontrolo da diabetes.

Este estudo permitiu comprovar a importância que tem os programas de educação para a saúde, sendo uma potente ferramenta de apoio à capacitação da população em estudo, promovendo assim o conhecimento e a capacidade para o autocontrolo das atividades de autocuidado relacionadas com a diabetes, contribuindo também para o aumento dos níveis de literacia em saúde na diabetes e para a maximização da qualidade de vida que será evidenciada a médio e longo prazo com ganhos em saúde.

Consideramos que focar a intervenção dos enfermeiros, nomeadamente do enfermeiro especialista em enfermagem comunitária no paradigma da promoção/prevenção, dará fortes contributos quer para a prevenção quer para o controlo das doenças crónicas e muito particularmente na diabetes mellitus.

Limitações do estudo: tamanho da amostra e centrada num só contexto

\section{Referências}

1. Observatório Nacional da Diabetes. Factos e Números 2015: Relatório Nacional da Diabetes [Internet]. Lisboa: Sociedade Portuguesa de Diabetologia; 2016 dez. [citado 2018 jan. 30]. Disponível na: www. https://www.sp d.pt/images/bolsas/dfn2015.pdf

2. Instituto Nacional de Estatística. Censos 2011. [Internet]. Lisboa: Instituto Nacional de Estatística; 2011 [citado 18 fev. 2018]. Disponível em: www.http://censos.ine.pt/xportal/xmain?xpid=CENSOS\&xpgid=censos2011_ apresentacao

3. Sousa MR, Peixoto MJ, Martins T. Satisfação do doente diabético com os cuidados de enfermagem: Influência na adesão ao regime terapêutico. Rev Ref. Dez [Internet]. 2008 [citado 18 fev. 2018];II série(8):59-67. Disponível em: http://www.index-f.com/referencia/2008pdf/8-5967.pdf

4. Associação Protectora dos Diabéticos de Portugal. Diabetes tipo 2: um guia de apoio e orientação. Lisboa: Lidel; 2009.

5. Bastos FS. Adesão e gestão do regime terapêutico do diabético tipo 2: Participação das esposas no plano educacional [tese de mestrado]. [Porto]: Universidade do Porto, Faculdade de Medicina; 2004 [citado 2018 mar. 01]. Disponível em: https://hdl.handle.net/10216/9654 
6. Bastos F, Severo M, Lopes C. Propriedades Psicométricas da Escala de Autocuidado com a Diabetes Traduzida e Adaptada. Acta Méd Port [Internet]. 2007;20:11-20 [citado 2018 mar. 01]. Disponível em: http://files.jvilelas. webnode.pt/200000087-60b9161b27/ESCALA\%20DE\%20AUTOCUIDADO.pdf

7. Martins AC, Andrade IM. Cross-cultural adaptation and validation of the Portuguese version of the Newest Vital Sign. Rev Ref [Internet]. 2014 [citado 2018 mar. 03]; 3:75-83. Disponível em: http://dx.doi.org/10.12707/R III1399

8. Krikorian A. Standards of medical care in diabetics. J Am Diabet Assoc. 2016;39(Supl 1):S1-2.

9. International Diabetes Federation. IDF Diabetes Atlas. $7^{\mathrm{a}}$ ed. Brussels (Belgium): International Diabetes Federation; 2015.

10. Ministério da Saúde de Portugal. Prescrição e Determinação da Hemoglobina Glicada A1C. DireçãoGeral de Saúde. Norma No 033. Lisboa: Ministério da Saúde de Portugal; 2011. Disponível em: https://www.dgs.pt/directrizes-da-dgs/normas-e-circulares-normativas/norma-n-0332011-de-30092011atualizada-a-06122012-jpg.aspx

11. Ortiz L, Pérez B, González E, Martínez S, Quirarte N, Berry D. Self-Care Behaviors and Glycemic Control in Low-Income Adults in México With Type 2 Diabetes Mellitus May Have Implications for Patients of Mexican Heritage Living in the United States. Clin Nur Research [Internet]. 2016 [citado 2018 mar. 07];25(2):120-138. Dispinível em: https://journals.sagepub.com/doi/10.1177/1054773815586542

12. Pedro AR, Amaral $\mathrm{O}$, Escoval A. Literacia em saúde, dos dados à ação: tradução, validação e aplicação do European Health Literacy Survey em Portugal. Rev Port Saúde Pública [Internet]. 2016 [citado 2018 mar. 10];34(3):259-275. Disponível em: https://www.sciencedirect.com/science/article/pii/S0870902516300311

13. Saboga-Nunes L, Sorensen K, Pelikan J, Cunha M, Rodrigues E, Paixão E. Cross-Cultural Adaptation and Validation to Portuguese of the European Health Literacy Survey (HLS-EU-PT). Atenc Primaria. 2014;46(espec cong 1):13. http://www.elsevier.es/es-revista-atencion-primaria-27-pdf

14. Boavida J. Educação do Doente Terapêutico no Tratamento da Diabetes. Rev Port Cardiolog [Internet]. 2013 [citado 2018 ago. 12];32(supl. 1):32-34. Disponível em: https://www.revportcardiol.org/pt-pdf-S087025511 3700453

15. Torres H, Franco L, Stradioto M, Hortale V, Schall V. Avaliação estratégica de educação em grupo e individual no programa educativo em diabetes. Rev Saúde Pública [Internet]. 2009 [citado 2018 set. 12];43(2):291-298. Disponível em: http://www.scielo.br/pdf/rsp/v43n2/05.pdf

\section{Notas}

* Artigo original de pesquisa

Licencia Creative Commons CC BY 4.0

Como citar este artigo: Rua-Castro ME, Soares S. Literacia em saúde e o autocuidado e autocontrolo no idoso com diabetes tipo 2. Investig Enferm Imagen Desarr. 2020;22. https://doi.org/10.11144/Javeriana.ie22.lsaa 